\title{
Melatonin ameliorates myocardial apoptosis by suppressing endoplasmic reticulum stress in rats with long-term diabetic cardiomyopathy
}

\author{
FANG-YUAN XIONG ${ }^{1 *}$, SONG-TAO TANG ${ }^{2 *}$, HUAN SU $^{1}$, HAI-QIN TANG ${ }^{3}$, \\ PIN JIANG ${ }^{3}$, QING ZHOU ${ }^{1}$, YUAN WANG ${ }^{1}$ and HUA-QING ZHU ${ }^{1}$ \\ ${ }^{1}$ Laboratory of Molecular Biology, Department of Biochemistry, Anhui Medical University; Departments of \\ ${ }^{2}$ Endocrinology and ${ }^{3}$ Geriatrics, The First Affiliated Hospital of Anhui Medical University, Hefei, Anhui 230032, P.R. China
}

Received November 11, 2016; Accepted August 24, 2017

DOI: $10.3892 / \mathrm{mmr} .2017 .7841$

\begin{abstract}
The effects of melatonin (MLT), which exerts cardioprotective effects against myocardial apoptosis, in long-term diabetic cardiomyopathy are not currently well defined. The present study aimed to investigate how MLT protects the heart through modulating myocardial apoptosis in rats with type 2 diabetes mellitus (DM). In total, 36 rats were randomly divided into three groups, including control $(n=12)$, DM $(n=12)$ and DM + MLT $(n=12)$ groups. The results demonstrated that, in DM rats, a significant increase was observed in the serum fasting blood glucose and lipid levels, in addition to insulin resistance and cardiac dysfunction, which were attenuated in DM rats treated with MLT. Additionally, cellular apoptosis in rats with DM was increased, and the expression of Bcl-2 was downregulated, while levels of Bcl-2-associated X and caspase- 3 were upregulated, and these observations were reversed by MLT, as determined by TUNEL and western blot analysis, respectively. As increased endoplasmic reticulum (ER) stress induced by hyperglycemia is reported to be a factor for apoptosis, the present study also determined the expression of proteins associated with ER stress in cardiac tissues following MLT treatment by western blotting. The results further indicated that MLT decreased the expression of ER stress hallmarks, including CCAAT/enhancer-binding protein homologous protein, glucose-regulated protein 78, protein kinase RNA-like endoplasmic reticulum kinase (PERK)
\end{abstract}

Correspondence to: Professor Hua-Qing Zhu or Professor Yuan Wang, Laboratory of Molecular Biology, Department of Biochemistry, Anhui Medical University, 81 Meishan Road, Hefei, Anhui 230032, P.R. China

E-mail: aydzhq@126.com

E-mail: aydesm-1@163.com

${ }^{*}$ Contributed equally

Key words: diabetic cardiomyopathy, melatonin, endoplasmic reticulum stress, apoptosis and activating transcription factor $6 \alpha$ in cardiac tissues. In conclusion, the results of the present study indicate that MLT may protect heart by ameliorating cardiac ER stress-induced apoptosis in diabetic cardiomyopathy.

\section{Introduction}

Type 2 diabetes mellitus (DM) is a disease that is associated with high morbidity and mortality due to various diabetic complications (1). Diabetic cardiomyopathy, a severe diabetic complication, refers to ventricular dysfunction independent of alterations in blood pressure and coronary artery disease $(2,3)$. In the past decades, numerous studies have investigated the mechanisms involved in diabetic cardiomyopathy (4-7). At present, of the various etiologies that have been reported to be associated the pathogenesis of diabetic cardiomyopathy, the apoptosis of cardiomyocytes is considered to be one of the primary causes of diabetic cardiomyopathy. Therefore, attenuation of cardiomyocyte apoptosis has been investigated as a potential therapeutic strategy for diabetic cardiomyopathy $(8,9)$.

The endoplasmic reticulum (ER) has important roles in the regulation of $\mathrm{Ca}^{2+}$ homoeostasis, and protein synthesis and folding (10). ER stress, which is characterized by a perturbation in the $\mathrm{Ca}^{2+}$ or the redox balance of the ER, is the primary cause of the accumulation of misfolded proteins $(11,12)$. At the initial stage of ER stress, a protective procedure termed unfolded protein response (UPR) is initiated in the ER (13), which is mediated by three transmembrane sensors, including protein kinase RNA-like endoplasmic reticulum kinase (PERK), activated transcription factor 6 (ATF-6) and inositol-requiring enzyme 1 (IRE1). Under non-stress conditions, the proteins are bound to the ER chaperone protein glucose-regulated protein 78 (GRP78). Upon accumulation of unfolded proteins in the ER, GRP78 becomes dissociated from these transducers to assist with the folding of the accumulated proteins (14). When ER stress is prolonged, cell death is triggered by activating CCAAT/enhancer-binding protein homologous protein (CHOP), phosphorylated (p)-eukaryotic initiation factor $2 \alpha$ (eIF2 $\alpha)$, caspase-12, caspase-9 and caspase-3 (15-19). Of these factors, CHOP, which exhibits low expression or is barely detected under non-stress conditions, 
promotes apoptosis by altering the $\mathrm{Bcl}-2 / \mathrm{Bcl}-2$-associated $\mathrm{X}$ (Bax) ratio and generating reactive oxygen species $(20,21)$. In addition, as previously described, cell apoptosis induced by ER stress is reported to be independent of death receptor and mitochondrial pathways (22). In animals with hyperglycemia, which is associated with the pathogenesis of diabetes, the level of ER stress was upregulated and associated with myocardial cell apoptosis (3-5). Therefore, the suppression of ER stress may serve as an effective strategy to alleviate myocardial cell apoptosis to protect the myocardial function.

Melatonin (MLT; N-acetyl-5-methoxytryptamine), an endogenous substance that is primarily secreted by the pineal body, has various properties, including antiapoptosis, anti-inflammatory and antitumor effects, in addition to roles in anti-oxidative stress (23-25). Previous studies have investigated the effects of MLT in the treatment of cardiovascular diseases (26-30). For example, Dominguez-Rodriguez et al (29) indicated that reduced MLT levels were associated with the development of heart failure in patients with hypertensive cardiomyopathy. Furthermore, convincing evidence also indicates that MLT is associated with protective effects against ER stress $(30,31)$.

Studies have previously investigated the role of MLT in the protection of cardiomyopathy (26-29). However, the mechanisms underlying these protective effects on diabetic cardiomyopathy are not currently well defined. The present study was designed to investigate whether MLT may relieve myocardial apoptosis in long-term diabetic cardiomyopathy by targeting ER stress.

\section{Materials and methods}

Experimental animals. All experimental protocols on animals were performed in compliance with and approved by the Ethics Committee of Anhui Medical University (Hefei, China). A total of 36 male Sprague-Dawley rats (6 weeks old; weight, 160-200 g) were purchased from the Experimental Animal Center of Anhui Medical University and raised in a specific pathogen free environment. After one week of adaptation, rats were randomized to control $(n=12), D M(n=12)$ and $\mathrm{DM}+\mathrm{MLT}(\mathrm{n}=12)$ groups. All rats were allowed free access to food and water and were kept in conditions of $22-24^{\circ} \mathrm{C}$ with a relative humidity of $40-70 \%$ on a $12 \mathrm{~h}$ light-dark cycle. All rats were fed a high-fat diet containing $2 \%$ cholesterol, $10 \%$ lard and $88 \%$ normal diet, apart from rats in the control group that were fed a normal diet. Intraperitoneal glucose tolerance test (IPGTT) and intraperitoneal insulin tolerance test (IPITT) were performed at week 8 (see below for specific steps). At week 9, control rats were injected with $1 \mathrm{~mol} / \mathrm{l}$ sodium citrate saline buffer (Beijing Chemical Reagent Co., Ltd, Beijing, China), while the rats in the DM and DM + MLT groups were subject to intraperitoneal administration of streptozocin (STZ; $25 \mathrm{mg} / \mathrm{kg}$ dissolved in $20 \mathrm{mM}$ sodium citrate saline buffer; Sigma-Aldrich; Merck KGaA, Darmstadt, Germany) once (26). The blood glucose levels were tested 1 week after the streptozocin injection. Animals with glucose levels $\geq 11.1 \mathrm{mmol} / 1$ were considered to be diabetic. Rats in the DM + MLT group subsequently received $10 \mathrm{mg} / \mathrm{kg} /$ day MLT (Institute of Clinical Pharmacology of Anhui Medical University, Hefei, China) by gavage for 24 weeks. The control and DM rat groups were treated with intragastric saline administration. At the end of week 33, animals were starved overnight and anesthetized using a $10 \%$ chloral hydrate solution $(300 \mathrm{mg} / \mathrm{kg}$; intraperitoneal injection). Cardiac function was evaluated under anesthesia. Blood samples were collected from the abdominal aorta. After the hearts from each rat were weighed and sliced, the heart weight/body weight (HW/BW) was calculated and the left ventricle (LV) was rapidly dissected and cut into two pieces. One piece was immediately frozen in liquid nitrogen and stored at $-80^{\circ} \mathrm{C}$ for protein analysis and the other was fixed with $10 \%$ neutral formalin for 6-8 days at room temperature for histological examination.

IPGTT. After a $12 \mathrm{~h}$ fast, all rats were intraperitoneally administrated with glucose $(1 \mathrm{~g} / \mathrm{kg}$ bodyweight dissolved in a sodium chloride injection; Sinopharm Chemical Reagent Co., Ltd, Shanghai, China). Blood glucose concentrations were detected using a blood glucose meter through a vein in the tail at 0,15 , 30,60 and $120 \mathrm{~min}$.

IPITT. After $12 \mathrm{~h}$ of abrosia, insulin (1I U/kg bodyweight dissolved in a sodium chloride injection; Novolin 30R, Novo Nordisk, Beijing, China) was intraperitoneally injected into rats. Then blood glucose concentrations were detected through tail venous blood 0, 15, 30, 60 and $120 \mathrm{~min}$.

Cardiac function testing. Cardiac function was measured under constant flow and constant pressure (32). Briefly, a specific indwelling needle was intubated to the left ventricle through via the right common carotid artery, which was connected to a force-displacement transducer to record tension and heart rate. Left ventricular end-diastolic pressure (LVEDP), left ventricular systolic pressure (LVSP), maximal rate of increase of left ventricular pressure $(+\mathrm{dp} / \mathrm{dtmax})$ and maximal rate of decrease of left ventricular pressure (-dp/dtmax) as the rate of contraction and relaxation were calculated using a BL-420 biological function experimental system (Chengdu Techman Software Co., Ltd., Chengdu, China).

Serum biochemical analysis. Blood samples were collected from the abdominal aorta and left for $\sim 1 \mathrm{~h}$ at room temperature until it is completely coagulated. The samples were then centrifuged at $1,000 \mathrm{x} \mathrm{g}$ for $10 \mathrm{~min}$ at room temperature, and serum was obtained and kept at $-80^{\circ} \mathrm{C}$. Serum fasting blood glucose (FBG), total cholesterol (TC), low-density lipoprotein (LDL) cholesterol, triglyceride (TG) and insulin levels were determined using commercially available spectrophotometric assay kits (Beijing BHKT Clinical Reagent Co., Ltd., Beijing, China), according to the manufacturer's protocol. The insulin sensitivity index was calculated using the following formula: 1 / (FBG x fasting insulin).

Histology. Formalin-fixed, paraffin-embedded myocardial tissue samples were cut into $6-\mu$ m-thick serial sections and stained by hematoxylin for $6 \mathrm{~min}$ and eosin for $2 \mathrm{~min}$ (H\&E) or Masson's trichrome staining at room temperature. Masson's trichome staining was performed to detect myocardial fibrosis by sequential addition of Bouin's, Weigert's and Biebrich solutions for $5 \mathrm{~min}$ each according manufacturer's protocol (Masson trichrome stain kit; cat. no. BSBA-4079A; 
Beijing Zhongshan Golden Bridge Biotechnology Co., Ltd., Beijing, China). Morphological analysis was performed using light microscopy and the mean optical density values for collagen levels were measured using a JD-801 pathological image analysis system (version 2013; Jiangsu JEDA Science-Technology Development Co., Ltd., Nanjing, China).

Apoptosis assays. Myocardial apoptosis was detected in 6- $\mu \mathrm{m}$-thick serial sections of myocardial tissue samples using a One Step TUNEL Apoptosis assay kit (Beyotime Institute of Biotechnology, Haimen, China) following their fixation with $10 \%$ formalin and embedding in paraffin. TUNEL staining was performed according to the manufacturer's protocol. TUNEL-positive cells, which exhibited green nuclear staining, were observed using a Leica fluorescence microscope (DM4000). All cells with DAPI and/or TUNEL staining were counted within five randomly selected fields in a blinded manner. The index of apoptosis was expressed as the ratio of positively stained apoptotic myocytes to the total number of myocytes counted.

Immunohistochemistry analysis. Formalin-fixed, paraffin-embedded 6- $\mu \mathrm{m}$-thick cardiomyocyte sections were used for immunohistochemical staining. The slides were deparaffinized by gradient concentration of ethanol (anhydrous ethanol, then 95, 80 and 70\% ethanol), rehydrated, placed in citric acid buffer $(0.01 \mathrm{~mol} / \mathrm{l} ; \mathrm{pH}=6.0$; Beijing Zhongshan Golden Bridge Biotechnology Co., Ltd.) to boil for $10 \mathrm{~min}$ for antigen retrieval, then cooled to room temperature. The slides were blocked with $3 \% \mathrm{H}_{2} \mathrm{O}_{2}$ solution for 20 min at room temperature and washed with phosphate buffer solution $(0.01 \mathrm{~mol} / \mathrm{l}, \mathrm{pH}=7.4$; Beijing Zhongshan Golden Bridge Biotechnology Co., Ltd.). Primary antibodies against caspase-3 (1:1,000; cat\# 9662; Cell Signaling Technology, Inc., Danvers, MA, USA) were incubated with the sections overnight at $4^{\circ} \mathrm{C}$. Subsequently, the slides were washed in Tris-buffered saline (TBS containing $10 \mathrm{mM} / 1$ Tris $\mathrm{HCl}$ and $0.85 \% \mathrm{NaCl} ; \mathrm{pH}=7.2$ ) and incubated with anti-rat IgG biotinylated antibody and horseradish peroxidase-conjugated streptavidin (Universal IHC two-step test kit; PV-6000; Beijing Zhongshan Golden Bridge Biotechnology Co., Ltd.) according to the manufacturer's protocol, for 10-20 min at room temperature. Finally, the samples were incubated with 3,3'-diaminobenzidine (1:20) for $6 \mathrm{~min}$ as the substrate and counterstained with hematoxylin for $3 \mathrm{~min}$ at room temperature. Caspase-3-positive cells were observed by a light microscope and the mean optical density values were measured using JD-801 pathological imaging analysis system (version 2013).

Western blotting. The protein expression of the ER stress proteins, GRP78, CHOP, ATF-6 $\alpha$, PERK, IRE1 $\alpha$ and caspase-12, and the apoptotic markers Bax, Bcl-2, caspase-3 and caspase- 9 , were assessed by western blotting analysis . Cardiac muscle tissue was sheared and ground with protein lysis solution (5X protein lysis solution: $21.75 \mathrm{~g} 150 \mathrm{mmol} / \mathrm{l}$ $\mathrm{NaCl}, 5 \mathrm{ml} \mathrm{1 \%}$ Triton-X100, $0.5 \mathrm{~g} \mathrm{0.1 \%} \mathrm{SDS} \mathrm{and} 50 \mathrm{mmol} / \mathrm{l}$ Tris- $\mathrm{HCl}$, the volume was made up to $500 \mathrm{ml}$ with ultrapure water) into homogenate on the ice. The $5 \mathrm{X}$ protein lysis solution, physiological saline, phenylmethylsulfonyl fluoride (cat. no. ST505; Beyotime Institute of Biotechnology) and leupeptin (cat. no. L2884; Sigma-Aldrich; Merck KGaA) were used in the ratio 200:800:1:1. The homogenate was centrifuged at $14,000 \mathrm{x} \mathrm{g}$ for $15 \mathrm{~min}$ at $4^{\circ} \mathrm{C}$. Then the supernatant fluid was collected, and protein concentration was determined by BCA. Proteins $(50 \mu \mathrm{g})$ were separated by $8-15 \%$ SDS-PAGE and electrophoretically transferred onto polyvinylidene fluoride membranes. Following blocking with 5\% non-fat dry milk for $2 \mathrm{~h}$ at room temperature, the membranes were washed with TBS-Tween-20 [150 mM NaCl, $50 \mathrm{mM}$ Tris ( $\mathrm{pH}=7.5)$ and $0.1 \%$ Tween-20] and incubated with primary antibodies against caspase-9 (1:1,000; cat\# sc-133109), caspase-3 (1:1,000; cat\# sc-373730), Bcl-2 (1:500; cat\# sc-23960), Bax (1:500; cat\# sc-20067), GRP78 (1:1,000; cat\# sc-376768), CHOP (1:1,000; cat\# sc-71136), PERK (1:50; cat\# sc-9477), ATF-6 $\alpha$ (1:500; cat\# sc-22799), IRE1 $\alpha$ (1:1,000; cat\# sc-10510), caspase-12 (1:500; cat\# sc-21747) and $\beta$-actin (1:1,000; cat\# sc-130300) overnight at $4^{\circ} \mathrm{C}$. All primary antibodies were obtained from Santa Cruz Biotechnology, Inc. (Dallas, TX, USA). The bound antibody was visualized with horseradish peroxidase-conjugated secondary antibodies (goat anti-mouse IgG; cat. no. AP124P; 1:1,000, goat anti-rabbit IgG, cat. no. AP132P, 1:2,000 and rabbit anti-goat IgG; cat. no. AP106P; 1:4,000; EMD Millipore, Billerica, MA, USA) for $2 \mathrm{~h}$ at room temperature. The bands were detected using an enhanced chemiluminescence kit (BeyoECL Plus; Beyotime Institute of Biotechnology) and rapidly exposed to an autoradiography film (Amersham Hyperfilm ECL; GE Healthcare Life Sciences, Little Chalfont, UK) to detect light emission by a nonradioactive method. The values were quantified using Quantity One software (version, 4.6.2; Bio-Rad Laboratories, Inc., Hercules, CA, USA). $\beta$-actin antibody was used as the internal reference.

Statistical analysis. Statistical analysis was performed using SPSS 18.0 software (SPSS, Inc., Chicago, IL, USA). Each experiment was repeated $\geq 3$ times. Data are presented as the mean \pm standard deviation. The differences among the groups were evaluated using one-way analysis of variance followed by the Student-Newman-Keuls method. $\mathrm{P}<0.05$ was considered to indicate a statistically significant difference.

\section{Results}

IPGTT and IPITT results. After week 8, IPGTT and IPITT results demonstrated that the blood glucose levels in DM rats were significantly higher compared with those in control rats at baseline and at 15, 30, 60 and 120 min time points $(\mathrm{P}<0.05$; Fig. 1).

Myocardial dysfunction in diabetic models. When investigating cardiac function at the end of week 33 , the results demonstrated that LVSP, $+\mathrm{dp} / \mathrm{dtmax}$ and $-\mathrm{dp} / \mathrm{dtmax}$ were significantly decreased, while the LVEDP was significantly increased, in DM rats compared with control rats $(\mathrm{P}<0.05$; Fig. 2). These results indicate that long-term hyperglycemia led to myocardial dysfunction. However, MLT improved cardiac function significantly compared with DM rats without MLT treatment $(\mathrm{P}<0.05$; Fig. 2$)$.

Basic parameters in diabetic models. After 24 weeks of MLT treatment, FBG, TC, LDL and TG levels, and HW/BW 
A

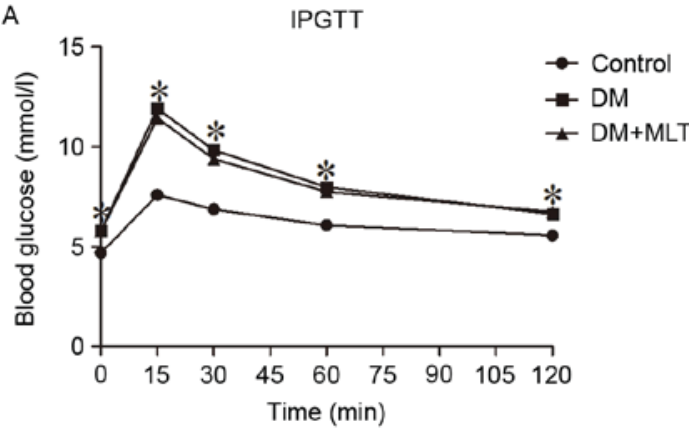

B

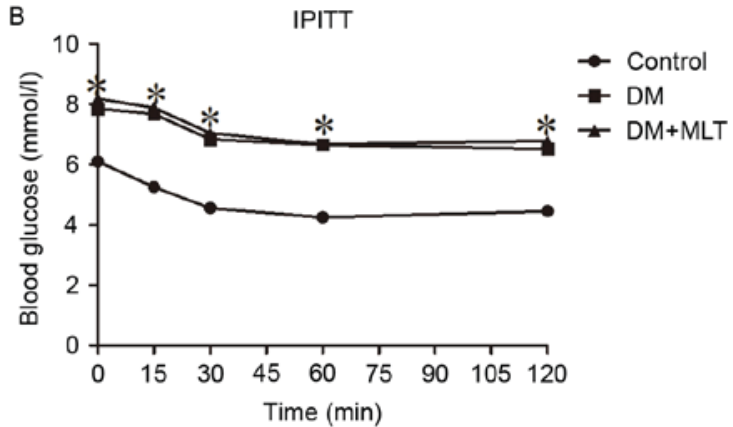

Figure 1. IPGTT and IPITT results at week 8 after a high-fat or normal diet. (A) Blood glucose levels during IPGTT. (B) Blood glucose levels during IPITT. Data are presented as the mean \pm standard deviation, $\mathrm{n} \geq 6$ per group. ${ }^{*} \mathrm{P}<0.05$ vs. control group. IPGTT, intraperitoneal glucose tolerance test; IPITT, intraperitoneal insulin tolerance test; DM, diabetes mellitus; MLT, melatonin.
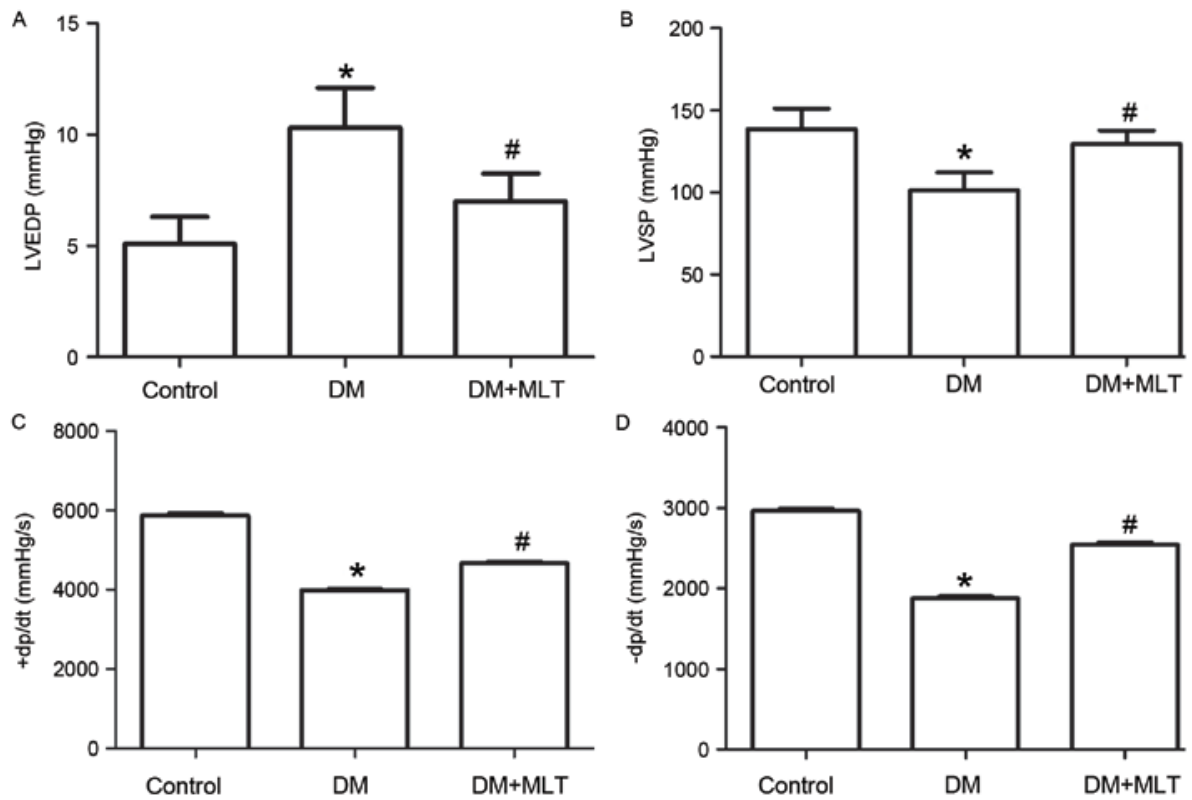

Figure 2. Cardiac function of DM rats with or without MLT treatment. The contractile function of rat hearts was determined. Alterations in (A) LVEDP, (B) LVSP, (C) $+\mathrm{dp} / \mathrm{dtmax}$ and (D) -dp/dtmax were examined. Data are presented as the mean + standard deviation, $\mathrm{n} \geq 6$ per group. "P<0.05 vs. control group; ${ }^{\text {"P }}<0.05$ vs. DM group. DM, diabetes mellitus; MLT, melatonin; LVEDP, left ventricular end-diastolic pressure; LVSP, left ventricular systolic pressure; + dp/dtmax, maximal rate of increase of left ventricular pressure; -dp/dtmax, maximal rate of decrease of left ventricular pressure.

and insulin resistance, were significantly higher in DM rats compared with control rats $(\mathrm{P}<0.05$; Table I). However, MLT therapy reduced FBG, lipid levels and the HW/BW (Table I). Additionally, MLT marginally reduced insulin resistance, although this change was not significant (Table I).

Morphological characteristics. The morphological characteristics of the myocardial lesions were examined by $\mathrm{H} \& \mathrm{E}$ (Fig. 3A) and Masson's trichome staining (Fig. 3B and C). Compared with the control rats, myocardial fibers were arranged irregularly and the staining was uneven in the rats of the DM group (Fig. 3A). MLT treatment improved the structural impairments of the myocardium and the disarrangement of myocardial fibers was corrected (Fig. 3A). Masson's staining demonstrated that collagen content was significantly increased and thick collagen fibers formed complex network structures in the heart of DM rats $(\mathrm{P}<0.05$; Fig. $3 \mathrm{~B}$ and $\mathrm{C})$. However, the collagen content was significantly reduced in DM rats treated with MLT $(\mathrm{P}<0.05$, Fig. 3B and $\mathrm{C})$.
Cardiac apoptosis. TUNEL assay results demonstrated that the percentage of apoptotic myocardial cells was significantly increased in DM rats compared with the control rats $(\mathrm{P}<0.05$; Fig. 4). However, a significant decrease in the percentage of apoptotic myocardial cells was observed in DM rats treated with MLT (Fig. 4). Caspase-3 is the primary terminal cleavage enzyme in the process of cell apoptosis. In the present study, immunohistochemical analysis demonstrated that the expression of caspase-3 was markedly upregulated in the cardiac muscle tissues of DM rats compared with the control rats $(\mathrm{P}<0.05$; Fig. 5), while MLT treatment reduced DM-induced upregulation of caspase-3 ( $\mathrm{P}<0.05$; Fig. 5).

Measurement of relative protein expression levels. The expression of caspase-3, caspase-9 and the proapoptotic protein Bax were significantly upregulated in the myocardium of DM rats compared with control rats, while the expression of the antiapoptotic protein Bcl-2 was significantly downregulated $(\mathrm{P}<0.05$; Fig. 6). These alterations were reversed in DM rats treated with 
Table I. Basic parameters of rats in different experimental groups at the end of 33 weeks.

\begin{tabular}{lrrc}
\hline Parameter & Control (n=6) & DM $(\mathrm{n}=6)$ & DM + MLT $(\mathrm{n}=8)$ \\
\hline FBG, mmol/1 & $4.75 \pm 0.38$ & $19.27 \pm 1.03^{\mathrm{a}}$ & $16.59 \pm 1.45^{\mathrm{a}, \mathrm{b}}$ \\
TC, $\mathrm{mmol} / \mathrm{l}$ & $2.53 \pm 0.08$ & $8.05 \pm 1.54^{\mathrm{a}}$ & $5.25 \pm 0.43^{\mathrm{a}, \mathrm{b}}$ \\
LDL, mmol/1 & $1.14 \pm 0.09$ & $6.12 \pm 1.96^{\mathrm{a}}$ & $3.81 \pm 0.48^{\mathrm{a}, \mathrm{b}}$ \\
TG, $\mathrm{mmol} / \mathrm{l}$ & $1.15 \pm 0.06$ & $5.63 \pm 0.77^{\mathrm{a}}$ & $4.08 \pm 0.48^{\mathrm{a}, \mathrm{b}}$ \\
Insulin, $\mu \mathrm{IU} / \mathrm{ml}$ & $11.40 \pm 2.15$ & $34.79 \pm 7.97^{\mathrm{a}}$ & $30.30 \pm 6.22^{\mathrm{a}}$ \\
Ln (ISI) & $-3.97 \pm 0.20$ & $-6.48 \pm 0.23^{\mathrm{a}}$ & $-6.04 \pm 0.42^{\mathrm{a}, \mathrm{b}}$ \\
HW/BW (x $\left.10^{-2}\right)$ & $0.23 \pm 0.02$ & $0.35 \pm 0.04^{\mathrm{a}}$ & $0.29 \pm 0.04^{\mathrm{a}, \mathrm{b}}$ \\
\hline
\end{tabular}

ISI $=1 /($ FBG $x$ fasting insulin). Data are presented as the mean \pm standard deviation. ${ }^{\mathrm{a}} \mathrm{P}<0.05$ vs. control group; ${ }^{\mathrm{b}} \mathrm{P}<0.05$ vs DM group. DM, diabetes mellitus; MLT, melatonin; FBG, fasting blood glucose; TC, total cholesterol; LDL, low-density lipoprotein; TG, triglyceride; ISI, insulin sensitivity index; HW, heart weight; BW, body weight.

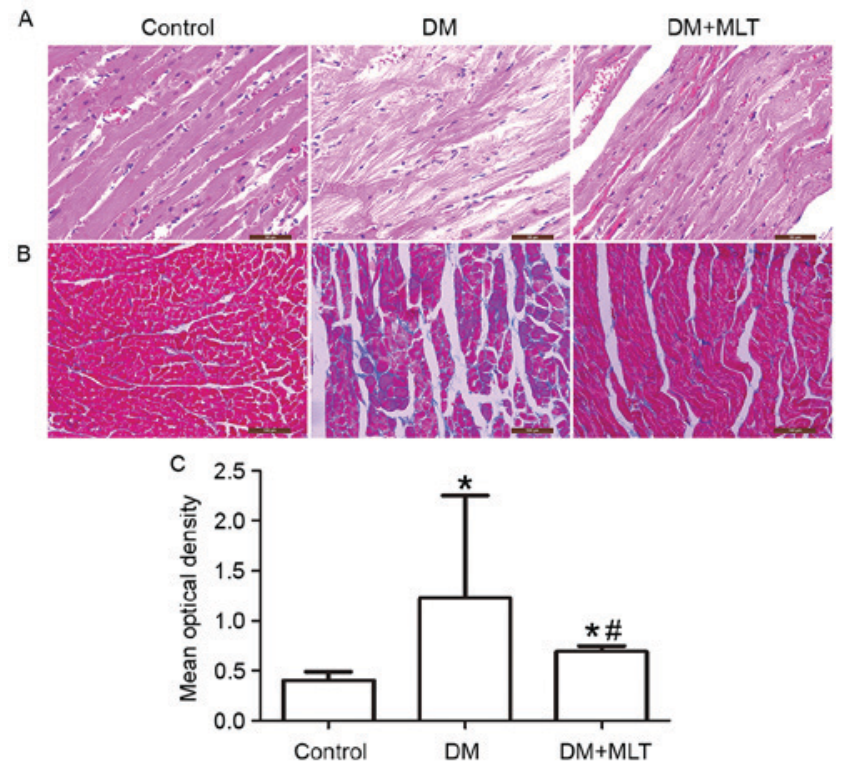

Figure 3. MLT relieved damage in the hearts of DM rats. (A) Hematoxylin and eosin stained sections of myocardium from different treatment groups. Magnification, x200. (B) Myocardium sections were stained by Masson's trichome staining. Magnification, x200. (C) Mean optical density of Masson's trichome stained sections indicates the content of the collagen. Data are presented as the mean + standard deviation, $n \geq 6$ per group. ${ }^{*} \mathrm{P}<0.05$ vs. control, ${ }^{\text {} P} \mathrm{P}<0.05 \mathrm{DM}+\mathrm{MLT}$ group vs. DM group. DM, diabetes mellitus; MLT, melatonin; SD, standard deviation.

MLT (P<0.05; Fig. 6). Furthermore, in rats in the DM group, the protein expression of GRP78, PERK, ATF- $6 \alpha$, caspase-12 and $\mathrm{CHOP}$ was significantly increased in the myocardium compared with control rats $(\mathrm{P}<0.05$; Fig. 7). However, in DM rats that received MLT treatment, the expression of these proteins was significantly downregulated $(\mathrm{P}<0.05 ;$ Fig. 7). Notably, although the expression of IRE1 $\alpha$ was significantly increased in DM rats compared with control rats $(\mathrm{P}<0.05)$, the expression of IRE1 $\alpha$ was not significantly reduced in DM rats with MLT treatment compared with the DM group (Fig. 7).

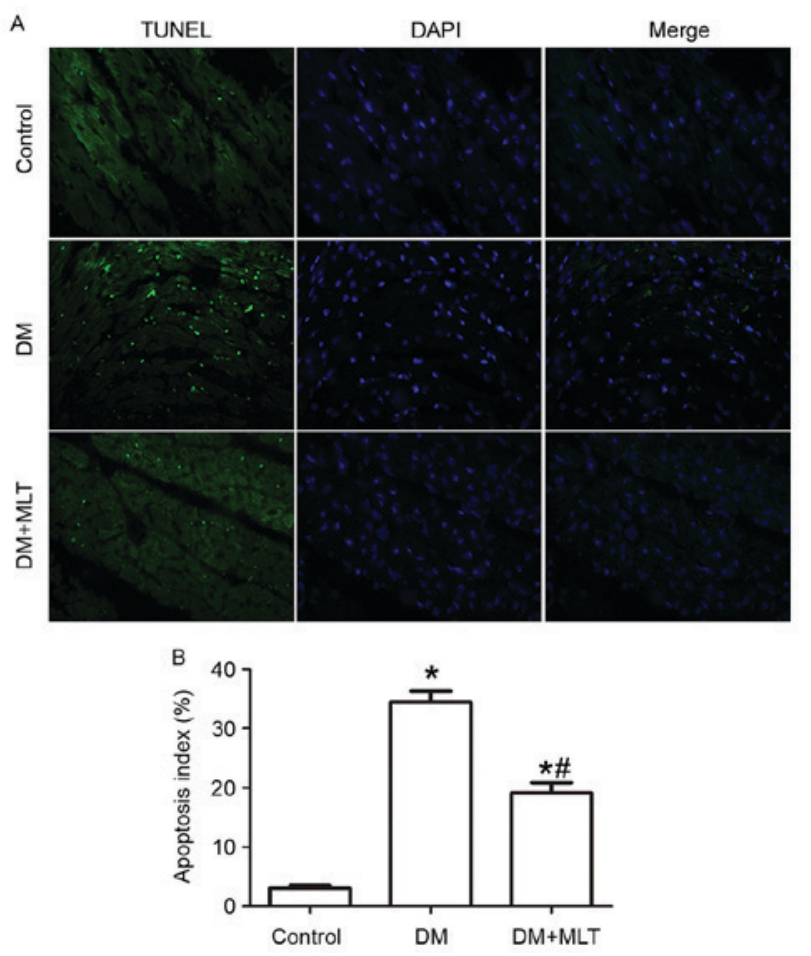

Figure 4. Melatonin ameliorated myocardial apoptosis in diabetic rats. (A) A representative photomicrographs of in situ detection of apoptotic myocytes by TUNEL staining under a magnification of x200. (B) The percentage of TUNEL-positive nuclei. 1: Control, 2: DM group, 3: DM + MLT group. Data are expressed as mean $\pm \mathrm{SD}, \mathrm{n} \geq 6$ per group. ${ }^{*} \mathrm{P}<0.05$ vs. control, ${ }^{\#} \mathrm{P}<0.05$ $\mathrm{DM}+\mathrm{MLT}$ group vs. DM group. TUNEL, terminal deoxynucleotidyl transferase dUTP nick end labeling; DM, diabetes mellitus; MLT, melatonin; SD, standard deviation.

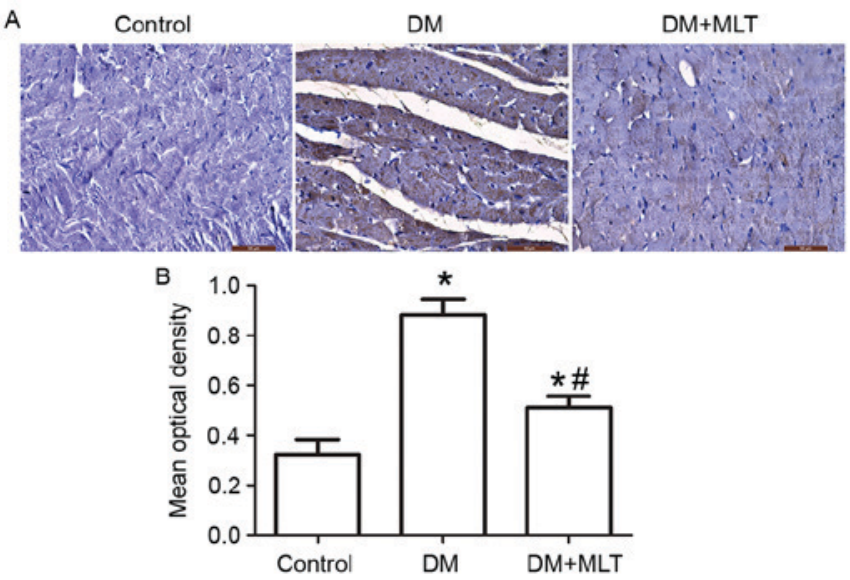

Figure 5. Effects of melatonin on Caspase-3 expression. (A) Immunohistochemistry showed the expression of Caspase- 3 in diabetic rats was increased (magnification x200). 1: Control, 2: DM group, 3: DM + MLT group. (B) Data are expressed as mean $\pm \mathrm{SD}, \mathrm{n} \geq 6$ per group. ${ }^{*} \mathrm{P}<0.05$ vs. control, ${ }^{\#} \mathrm{P}<0.05 \mathrm{DM}+\mathrm{MLT}$ group vs. DM group. DM, diabetes mellitus; MLT, melatonin; SD, standard deviation.

\section{Discussion}

The present study established a rat model of type 2 DM to investigate the therapeutic effects of MLT in diabetic cardiomyopathy. The results of this study indicate that MLT 

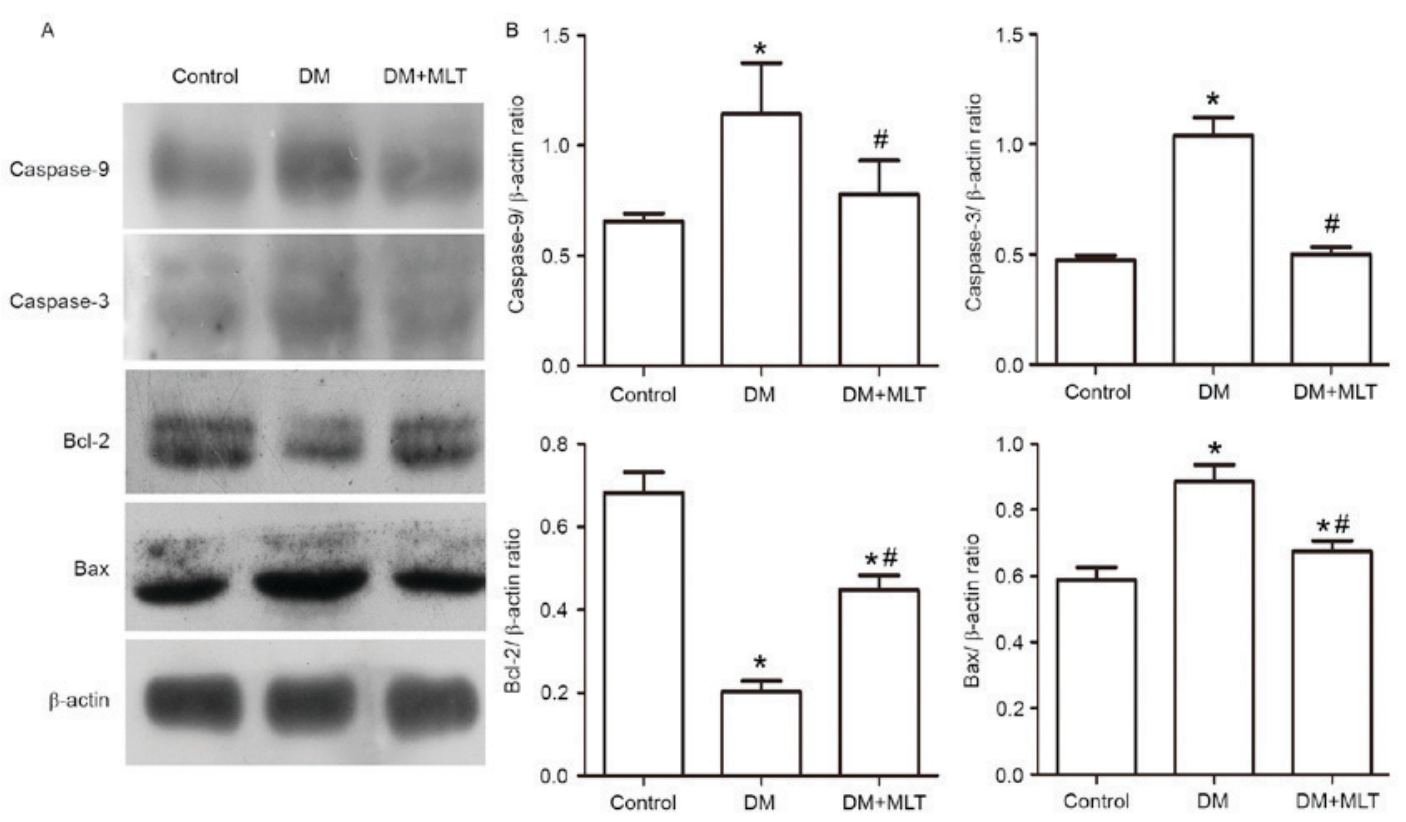

Figure 6. (A) Effect of melatonin on the expression of Caspase-9, Caspase-3, Bcl-2 and Bax in myocardial tissue. 1: Control, 2: DM group 3: DM + MLT group. (B) Data are expressed as mean $\pm \mathrm{SD}, \mathrm{n} \geq 6$ per group. ${ }^{*} \mathrm{P}<0.05$ vs. control, ${ }^{*} \mathrm{P}<0.05 \mathrm{DM}+\mathrm{MLT}$ group vs. DM group. DM, diabetes mellitus; MLT, melatonin; SD, standard deviation.
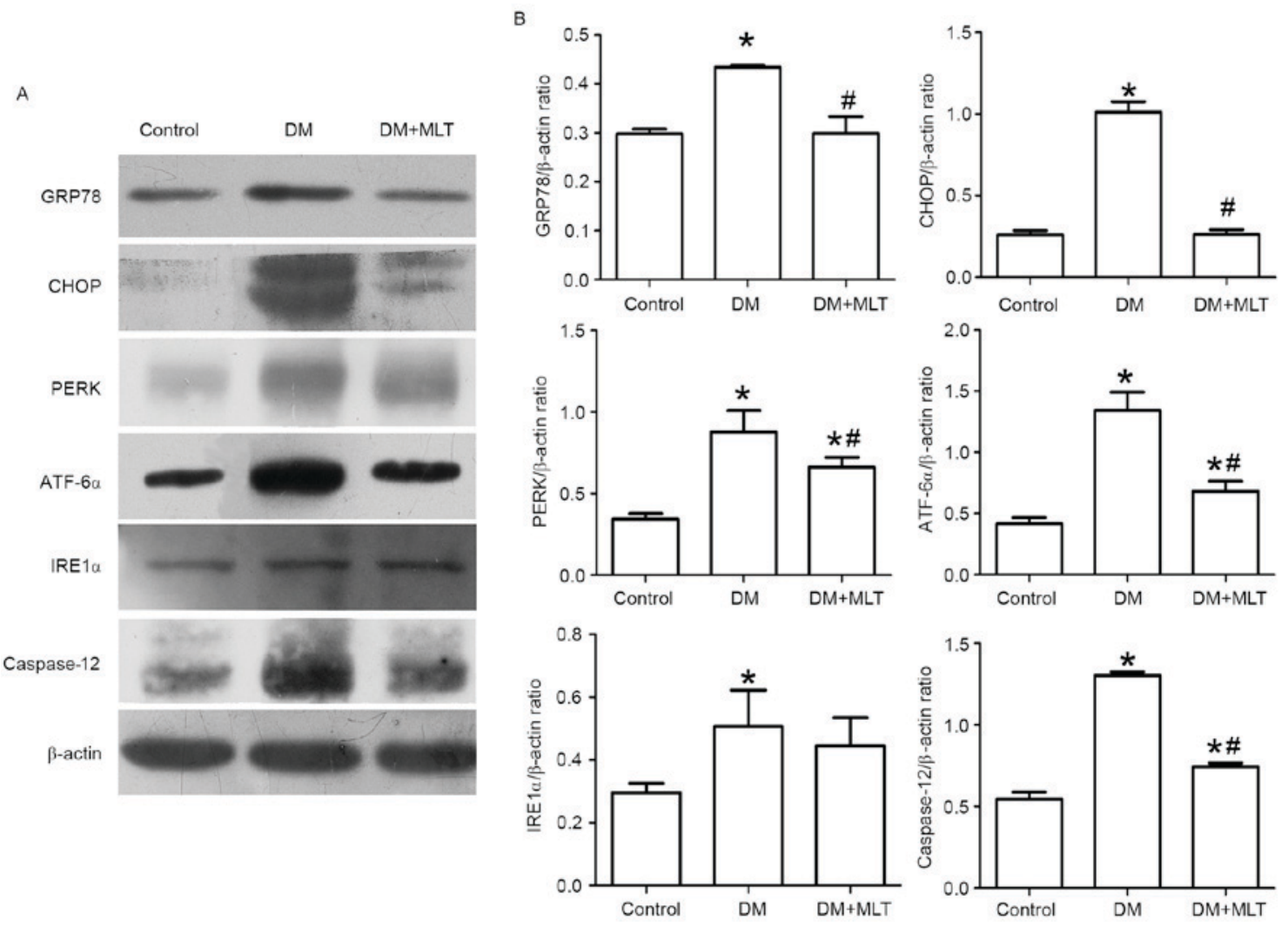

Figure 7. (A) Western blot was performed to measure GRP78, CHOP, PERK, ATF-6 $\alpha$, IRE1 $\alpha$ and Caspase-12 in myocardial tissue. 1: Control, 2: DM group, 3 : $\mathrm{DM}+$ MLT group. (B) Data are expressed as mean $\pm \mathrm{SD}, \mathrm{n} \geq 6$ per group. ${ }^{*} \mathrm{P}<0.05$ vs. control, ${ }^{*} \mathrm{P}<0.05 \mathrm{DM}+\mathrm{MLT}$ group vs. DM group. DM, diabetes mellitus; MLT, melatonin; SD, standard deviation.

may exert a protective effect on cardiomyopathy by suppressing ER stress.

Apoptosis is considered to be closely associated with the pathogenesis of diabetic cardiomyopathy (33). It has also been reported that cardiomyocytes undergo apoptosis in response to diabetic hyperglycemia, inflammation and ER stress (34). In addition, evidence indicates that the apoptosis of cardiomyocytes was increased in animals with DM (8). Furthermore, 
cardiomyocytes apoptosis is also the key initiating factor for cardiac dysfunction by stimulating cardiomyocyte hypertrophy and fibroblast proliferation. The most recognized marker proteins of apoptosis are Bax, caspase- 3 and the antiapoptotic protein $\mathrm{Bcl}-2$. Of these, caspase-3, a member of the caspase family that is involved in programmed cell death, is a frequently activated death protease that catalyzes the cleavage of key cellular proteins $(35,36)$. In the present study, TUNEL assays demonstrated increased apoptosis in cardiac myocytes of the DM group compared with the control group. In addition, the expression of caspase-3 and Bax were significantly upregulated in the DM group compared with control rats. With regards to the expression of $\mathrm{Bcl}-2$, an antiapoptotic member of the Bcl-2 family (37), significant downregulation was observed in the DM rats compared with the control group. Consistent with previous studies, the results of the current study indicate that apoptosis is elevated was increased in rats with diabetic myocardiopathy.

MLT has been reported to exert protective effects on the heart against myocardial infarction (38). Notably, it is able to prevent apoptosis in several biological processes, including the induction of interleukin release. In a recent study, Amin et al (28) reported that MLT ameliorated apoptosis through extrinsic and intrinsic pathways. In the present study, melatonin ameliorated the apoptosis of cardiomyocytes through upregulation of $\mathrm{Bcl}-2$, and downregulation of Bax and Caspase-3, compared with the DM group.

Various studies have focused on the mechanisms of regulating the apoptosis of cardiomyocytes in DM $(39,40)$. The identification of agents capable of interfering with apoptosis is clinically important to target the specific pathways that are activated during diabetic cardiomyopathy. ER stress may be a potential cause of apoptosis in diabetic cardiomyopathy. Previously, hyperglycemia and insulin resistance were reported to enhance ER stress in diabetic cardiomyocytes. Other than hyperglycemia, the diabetic myocardium may experience other adverse factors, including oxidative stress, hyperhomocysteinemia, hypoxia and lipid deposition, which may lead to ER stress (41). According to previous studies $(42,43)$, ER stress may induce apoptosis through a complex mechanism in which UPR-mediated signals have important roles in the initiation, commitment and execution of the process. The present study primarily focused on the function of PERK, CHOP and ATF- $6 \alpha$. Previous studies have reported that signaling via PERK and ATF- $6 \alpha$ contributed to the triggering of proapoptotic signals (42-44). Regarding the mechanism, PERK is not active under normal conditions, however, in the presence of ER stress, dissociation of GRP78 from PERK initiates the dimerization and autophosphorylation of the kinase, which activates PERK. Upon dissociation of GRP78, ATF-6 $\alpha$ is also activated, which moves to the nucleus and induces genes with an ER stress response element. Furthermore, downstream elements such as $\mathrm{CHOP}$ or c-Jun $\mathrm{N}$-terminal kinase may induce apoptosis in cells. The results of the current study demonstrated that the expression of PERK, CHOP and ATF- $6 \alpha$ in DM rats was downregulated when treated with MLT. On this basis, we hypothesized that MLT may attenuate the apoptosis of cardiomyocytes by suppressing the ER stress.

Previously, MLT was demonstrated to protect against the cardiac toxicity of doxorubicin in rats (45). Furthermore, MLT improved cardiovascular function and attenuated damage in the heart of rats with renovascular hypertension, although the exact mechanism remains to be established (46). In the present study, the results indicated that MLT reduced LVEDP, and increased LVSP, $+\mathrm{dp} / \mathrm{dt}$ and $-\mathrm{dp} / \mathrm{dt}$, compared with DM rats without MLT treatment. These results demonstrate that MLT may improve cardiac function DM rats.

In conclusion, ER stress has an important role in the activation of apoptosis in the streptozocin-induced diabetic rat model. The results of the present study may provide a novel therapeutic strategy for treating diabetic myocardial injury. The beneficial effects of MLT may, at least partially, occur via the PERK/ATF- $6 \alpha /$ CHOP pathway. Therefore, MLT may have potential for clinical use as an adjuvant therapy to treat diabetic cardiomyopathy. Further in vitro studies are required to fully elaborate the effect of MLT on diabetic ER stress.

\section{Acknowledgements}

The present study was supported by the National Natural Science Foundation of China (grant nos. 81570419, 81470568 and 81270372), the Anhui Provincial Natural Science Foundation (grant no. 1608085MH168) and the Research Project for Practice Development of National TCM Clinical Research Bases (grant no. JDZX2015133).

\section{References}

1. Gregg EW, Li Y, Wang J, Burrows NR, Ali MK, Rolka D, Williams DE and Geiss L: Changes in diabetes-related complications in the United States, 1990-2010. N Engl J Med 370: 1514-1523, 2014.

2. Duckworth WC: Hyperglycemia and cardiovascular disease. Curr Atheroscler Rep 3: 383-391, 2001.

3. Cicek FA, Toy A, Tuncay E, Can B and Turan B: Beta-blocker timolol alleviates hyperglycemia-induced cardiac damage via inhibition of endoplasmic reticulum stress. J Bioenerg Biomembr 46: 377-387, 2014.

4. Zhang X, Ma X, Zhao M, Zhang B, Chi J, Liu W, Chen W, Fu Y, Liu Y and Yin X: $\mathrm{H} 2$ and $\mathrm{H} 3$ relaxin inhibit high glucose-induced apoptosis in neonatal rat ventricular myocytes. Biochimie 108: 59-67, 2015.

5. Li Z, Zhang T, Dai H, Liu G, Wang H, Sun Y, Zhang Y and Ge Z: Endoplasmic reticulum stress is involved in myocardial apoptosis of streptozocin-induced diabetic rats. J Endocrinol 196: 565-572, 2008.

6. Sari FR, Watanabe K, Widyantoro B, Thandavarayan RA Harima M, Zhang S, Muslin AJ, Kodama M and Aizawa Y: Partial inactivation of cardiac 14-3-3 protein in vivo elicits endoplasmic reticulum stress (ERS) and activates ERS-initiated apoptosis in ERS-induced mice. Cell Physiol Biochem 26: $167-178,2010$.

7. Lakshmanan AP, Harima M, Suzuki K, Soetikno V, Nagata M, Nakamura T, Takahashi T, Sone H, Kawachi H and Watanabe K: The hyperglycemia stimulated myocardial endoplasmic reticulum (ER) stress contributes to diabetic cardiomyopathy in the transgenic non-obese type 2 diabetic rats: A differential role of unfolded protein response (UPR) signaling proteins. Int J Biochem Cell Biol 45: 438-447, 2013.

8. Cai L and Kang YJ: Cell death and diabetic cardiomyopathy. Cardiovasc Toxicol 3: 219-228, 2003.

9. Gao Q, Wang XM, Ye HW, Yu Y, Kang PF, Wang HJ, Guan SD and $\mathrm{Li} \mathrm{ZH}$ : Changes in the expression of cardiac mitofusin-2 in different stages of diabetes in rats. Mol Med Rep 6: 811-814, 2012.

10. Groenendyk J, Agellon LB and Michalak M: Coping with endoplasmic reticulum stress in the cardiovascular system. Annu Rev Physiol 75: 49-67, 2013.

11. Prins D and Michalak M: Endoplasmic reticulum proteins in cardiac development and dysfunction. Can J Physiol Pharmacol 87: 419-425, 2009. 
12. Zhou H and Liu R: ER stress and hepatic lipid metabolism. Front Genet 9: 112, 2014.

13. Senft D and Ronai ZA: UPR, autophagy, and mitochondria crosstalk underlies the ER stress response. Trends Biochem Sci 40: 141-148, 2015.

14. Zhang L, Lai E, Teodoro $\mathrm{T}$ and Volchuk A: GRP78, but not protein-disulfide isomerase, partially reverses hyperglycemia induced inhibition of insulin synthesis and secretion in pancreatic \{beta\}-cells. J Biol Chem 284: 5289-5298, 2009.

15. Kepp O, Semeraro M, Bravo-San Pedro JM, Bloy N, Buqué A, Huang X, Zhou H, Senovilla L, Kroemer G and Galluzzi L: eIF $2 \alpha$ phosphorylation as a biomarker of immunogenic cell death. Semin Cancer Biol 33: 86-92, 2015.

16. Scull CM and Tabas I: Mechanisms of ER stress-induced apoptosis in atherosclerosis. Arterioscler Thromb Vasc Biol 31 2792-2797, 2011

17. Sano R and Reed JC: ER stress-induced cell death mechanisms. Biochim Biophys Acta 1833: 3460-3470, 2013.

18. Boyce $\mathrm{M}$ and Yuan J: Cellular response to endoplasmic reticulum stress: A matter of life or death. Cell Death Differ 13: 363-373, 2006.

19. Wu L, Cai B, Zheng S, Liu X, Cai H and Li H: Effect of emodin on endoplasmic reticulum stress in rats with severe acute pancreatitis. Inflammation 36: 1020-1029, 2013

20. Araki E, Oyadomari S and Mori M: Endoplasmic reticulum stress and diabetes mellitus. Intern Med 42: 7-14, 2003.

21. Oyadomari S and Mori M: Roles of CHOP/GADD153 in endoplasmic reticulum stress. Cell Death Differ 11: 381-389, 2004

22. Nakagawa T, Zhu H, Morishima N, Li E, Xu J, Yankner BA and Yuan J: Caspase-12 mediates endoplasmic-reticulum-specific apoptosis and cytotoxicity by amyloid-beta. Nature 403: 98-103, 2000.

23. Hill SM, Frasch T, Xiang S, Yuan L, Duplessis T and Mao L: Molecular mechanisms of melatonin anticancer effects. Integr Cancer Ther 8: 337-346, 2009.

24. Mauriz JL, Collado PS, Veneroso C, Reiter RJ and González-Gallego J: A review of the molecular aspects of melatonin's anti-inflammatory actions: Recent insights and new perspectives. J Pineal Res 54: 1-14, 2013.

25. Galano A, Tan DX and Reiter RJ: Melatonin as a naturally against oxidative stress: A physiochemical examination. J Pineal Res 51: 1-16, 2011.

26. Yu L, Liang H, Dong X, Zhao G, Jin Z, Zhai M, Yang Y, Chen W, Liu J, Yi W, et al: Reduced silent information regulator 1 signaling exacerbates myocardial ischemia-reperfusion injury in type 2 diabetic rats and the protective effect of melatonin. J Pineal Res 59: 376-390, 2015.

27. Yang Y, Duan W, Jin Z, Yi W, Yan J, Zhang S, Wang N, Liang Z, Li Y, Chen W, et al: JAK2/STAT3 activation by melatonin attenuates the mitochondrial oxidative damage induced by myocardial ischemia/reperfusion injury. J Pineal Res 55 . 275-286, 2013.

28. Amin AH, El-Missiry MA and Othman AL: Melatonin ameliorates metabolic risk factors, modulates apoptotic proteins, and protects the rat heart against diabetes-induced apoptosis. Eur J Pharmacol 747: 166-173, 2015.

29. Dominguez-Rodriguez A, Abreu-Gonzalez P and Reiter RJ: The potential usefulness of serum melatonin level to predict heart failure in patients with hypertensive cardiomyopathy. Int J Cardiol 174: 415-417, 2014.
30. Hadj Ayed Tka K, Mahfoudh Boussaid A, Zaouali MA, Kammoun R, Bejaoui M, Ghoul Mazgar S, Rosello Catafau J and Ben Abdennebi H: Melatonin modulates endoplasmic reticulum stress and Akt/GSK3-beta signaling pathway in a rat model of renal warm ischemia reperfusion. Anal Cell Pathol (Amst) 2015: 635172,2015

31. Fernández A, Ordóñez R, Reiter RJ, González-Gallego J and Mauriz JL: Melatonin and endoplasmic reticulum stress: Relation to autophagy and apoptosis. J Pineal Res 59: 292-307, 2015.

32. Layland J, Cave AC, Warren C, Grieve DJ, Sparks E, Kentish JC, Solaro RJ and Shah AM: Protection against endotoxemia-induced contractile dysfunction in mice with cardiac-specific expression of slow skeletal troponin I. FASEB J 19: 1137-1139, 2005.

33. Ouyang C, You J and Xie Z: The interplay between autophagy and apoptosis in the diabetic heart. J Mol Cell Cardio 71: 71-80, 2014.

34. Liu Q, Wang S and Cai L: Diabetic cardiomyopathy and its mechanisms: Role of oxidative stress and damage. J Diabetes Investig 5: 623-634, 2014

35. Roos WP and Kaina B: DNA damage-induced cell death by apoptosis. Trends Mol Med 12: 440-450, 2006

36. Wang JY: DNA damage and apoptosis. Cell Death Differ 8: 1047-1048, 2001

37. Tsujimoto Y: Role of Bcl-2 family proteins in apoptosis: Apoptosomes or mitochondria? Genes Cells 3: 697-707, 1998

38. Acikel M, Buyukokuroglu ME, Aksoy H, Erdogan F and Erol MK: Protective effects of melatonin against myocardial injury induced by isoproterenol in rats. J Pineal Res 35: 75-79, 2003.

39. Sheu JJ, Chang LT, Chiang CH, Sun CK, Chang NK, Youssef AA, Wu CJ, Lee FY and Yip HK: Impact of diabetes on cardiomyocyte apoptosis and connexin43 gap junction integrity: Role of pharmacological modulation. Int Heart J 48: 233-245, 2007.

40. Ghosh S, Pulinilkunnil T, Yuen G, Kewalramani G, An D, Qi D, Abrahani A and Rodrigues B: Cardiomyocyte apoptosis induced by short-term diabetes requires mitochondrial GSH depletion. Am J Physiol Heart Circ Physiol 289: H768-H776, 2005.

41. Li Z, Zhang T, Dai H, Liu G, Wang H, Sun Y, Zhang Y and $\mathrm{Ge} Z$ : Involvement of endoplasmic reticulum stress in myocardial apoptosis of streptozocin-induced diabetic rats. J Clin Biochem Nutr 41: 58-67, 2007.

42. Sovolyova N, Healy S, Samali A and Logue SE: Stressed to death-mechanisms of ER stress-induced cell death. Biol Chem 395: 1-13, 2014.

43. Di Sano F, Ferraro E, Tufi R, Achsel T, Piacentini M and Cecconi F: Endoplasmic reticulum stress induces apoptosis by an apoptosome-dependent but Caspase 12-independent mechanism. J Biol Chem 281: 2693-2700, 2006.

44. Szegezdi E, Logue SE, Gorman AM and Samali A: Mediators of endoplasmic reticulum stress-induced apoptosis. EMBO Rep 7: 880-885, 2006

45. Xu MF, Ho S, Qian ZM and Tang PL: Melatonin protects against cardiac toxicity of doxorubicin in rat. J Pineal Res 31: 301-307, 2001.

46. Erşahin M, Sehirli O, Toklu HZ, Süleymanoglu S, Emekli-Alturfan E, Yarat A, Tatlidede E, Yeğen BC and Sener G: Melatonin improves cardiovascular function and ameliorates renal, cardiac and cerebral damage in rats with renovascular hypertension. J Pineal Res 47: 97-106, 2009. 